\title{
Description des lésions unguéales des doigts dans un service d'urgence
}

\author{
Finger Nail Injuries in a Trauma Center
}

\author{
S. Carmès \\ Reçu le 4 février 2018; accepté le 18 mai 2018 \\ (C) SFMU et Lavoisier SAS 2018
}

Résumé Introduction : Nous rapportons une étude épidémiologique des lésions unguéales vues dans un centre de traumatologie de la main pendant une période de 18 mois. Les traumatismes unguéaux représentaient $8 \%$ de tous les traumatismes des doigts.

Méthodes : Nous avons pris en charge de façon continue 187 patients dont les dossiers ont été revus rétrospectivement. Matériel et résultats : Cent trente-huit hommes (74\%), avec un âge moyen de 38,5 ans, et 49 femmes (26\%), d'âge moyen de 46,1 ans. La main droite était atteinte 80 fois ( $43 \%)$, la gauche 106 fois $(57 \%)$, et les données étaient manquantes dans un cas. Les patients étaient atteints sur leur main dominante dans $37 \%$ des cas. Le pouce était le doigt le plus souvent touché, 55 cas (29\%), puis le majeur (23\%), l'index (17\%), l'annulaire (15\%) et l'auriculaire (10\%). Dans $5 \%$ des cas, il $\mathrm{y}$ avait plusieurs doigts touchés, et le majeur était alors toujours atteint. Un mécanisme d'écrasement était la cause la plus fréquente $(44 \%)$, avec un doigt coincé dans une porte dans 42 cas ( $23 \%$ ) ou écrasé par un objet (22\%). Venaient ensuite les plaies franches, 55 cas (30\%). L'exploration des lésions a retrouvé 47 lésions isolées de la tablette unguéale (25\%), 103 lésions du lit de l'ongle (55\%), 14 lésions de la matrice \pm associées à une lésion du lit et 23 hématomes sous-unguéaux (12\%). Dans 134 cas (72\%), il existait une autre lésion associée à celle de l'ongle. Il s'agissait d'une lésion pulpaire dans 50 cas (27\%), d'une fracture de la phalange distale dans 29 cas $(16 \%)$, d'une lésion associant à une fracture de la phalange distale une lésion de la pulpe dans 49 cas (26\%) et d'une autre lésion du doigt ou de la main dans 22 cas (12\%). La lésion siégeait sur le même doigt dans 128 cas.

Conclusion : Les lésions unguéales sont fréquentes. Le médecin urgentiste doit connaître ces lésions et ne pas les sous-estimer, car dans plus de trois quarts des cas elles nécessitent un geste technique qui relève le plus souvent de la compétence de l'urgentiste.

\section{S. Carmès $(\bowtie)$}

Urgences Main Guadeloupe, clinique des Eaux-Claires,

ZAC Moudong Sud, F-97122 Baie-Mahault, France

e-mail : Sylvie.Carmes@wanadoo.fr
Mots clés Ongle $\cdot$ Traumatisme de l'ongle $\cdot$ Doigt de porte $\cdot$ Épidémiologie

Abstract Introduction: We report an epidemiological survey of nail injuries seen in a hand trauma center during a period of 18 months. Nail traumas represented $8 \%$ of all finger injuries.

Methods: We followed 187 patients whose files have been retrospectively reviewed.

Material and results: Thousand one hundred and thirtyeight males $(74 \%)$ with an average age of 38 years and 49 females $(26 \%)$ with an average age of 46 years. The right side hand was involved 80 times (43\%), the left 106 times $(57 \%)$, and the data was missing in one patient. Patients were injured on their dominant side in $37 \%$ of cases. The thumb was most frequently involved with 55 cases $(29 \%)$, then was the middle finger $(23 \%)$, the index $(17 \%)$, the ring finger $(15 \%)$, and the auricular $(10 \%)$. In $5 \%$ of the cases (10 patients) many fingers were involved and in that instance, the middle finger was always injured. A crushing mechanism was the most frequent cause (44\%) with a finger trapped in a door in 42 cases $(23 \%)$ and hit by another object $(22 \%)$, then a sharp injury (30\%). Surgical exploration found 47 isolated lesions of the nail plate (25\%), 103 nail bed lesions (55\%), 14 matrix ( \pm bed) lesions and 23 nail bed hematomas (12\%). In 134 cases (72\%), there was an associated lesion to the nail injury: a lesion of the pulp in 50 cases $(27 \%)$, a fracture of the distal phalanx (16\%), or an association of a pulp injury and a fracture of the distal phalanx (26\%). The associated lesion was in the finger or the hand in 22 cases $(12 \%)$. The associated lesion was on the same finger in 128 cases.

Conclusion: Nail injuries are frequent. The emergency physician must know these injuries and not underestimate them as more than $75 \%$ should be treated "surgically".

Keywords Nail traumatism · Door finger trap · Epidemiology $\cdot$ Nail injury 


\section{Introduction}

Les traumatismes de la main font partie des urgences les plus fréquentes accueillies dans un service d'urgence. Parmi celles-ci, les traumatismes de l'ongle sont très fréquents. Chez l'enfant, le doigt de porte représente entre 5 et $6 \%$ des traumatismes [1,2]. Un diagnostic lésionnel et/ou un traitement inadapté peut entraîner des séquelles fonctionnelles ou cosmétiques, alors qu'un traitement adapté donne habituellement de bons ou d'excellents résultats $[1,3,4]$. Nos connaissances épidémiologiques de ces lésions reposent sur des travaux maintenant anciens qui concernaient des populations urbaines nord-américaines. Zook et al. ont rapporté 299 lésions unguéales sur une période de cinq ans, et l'un des coauteurs a ensuite rapporté 30 cas $[5,6]$. Dans ces études, les lésions unguéales faisaient suite à un mécanisme d'écrasement. Il existait le plus souvent des lésions associées de la pulpe ou une fracture phalangienne, et le majeur était le doigt le plus atteint [5-7]. Travaillant au sein d'une population plus agricole et dans une île des caraïbes, notre expérience était un peu différente, ce qui nous a poussé à revoir nos dossiers.

\section{Patients et méthodes}

Tous les patients atteints d'une lésion de la main et arrivant aux urgences étaient dirigés dans un circuit court pour être pris en charge, dans la journée, par un urgentiste positionné dans une salle dédiée d'accueil et de tri des urgences de la main. Tous les patients arrivant dans cette unité étaient enregistrés dans un cahier avec le motif de leur prise en charge. Travaillant dans un hôpital d'adulte, nous n'avons pris en charge que des patients de plus de 15 ans. Ont également été exclues les amputations distales de doigt. Sur une période de 18 mois, nous avons recherché dans les dossiers des patients atteints de lésions unguéales : l'âge, le sexe, la main dominante, le(s) doigt(s) atteint(s) et le mécanisme des lésions. L'occupation professionnelle était également notée. Des radiographies systématiques pour tous les traumatismes de la main permettaient de dépister les lésions osseuses associées. Une éventuelle atteinte pulpaire était également recherchée.

Les lésions unguéales ont été classées selon la lésion anatomique principale en lésion isolée de la tablette, en hématome sous-unguéal, en lésion du lit unguéal ou en lésion de la matrice unguéale [8] (Fig. 1).

Nous n'avons pas différencié entre lésions simples et complexes du lit comme proposé par Zook et al. [5].

Bien qu'il s'agisse d'une étude épidémiologique, les principes du traitement réalisé dans notre salle d'urgence ont été les suivants, ce qui nous a permis de mieux décrire les lésions. Seuls les hématomes sous-unguéaux douloureux ont été traités. Les hématomes dont l'étendue faisait moins de $50 \%$ de la surface de la tablette ont été évacués par deux trous réalisés avec la pointe d'une aiguille. Pour les hématomes faisant plus de $50 \%$ de la surface de la tablette, sous anesthésie locale intrathécale [9], la tablette était retirée, et les lésions du périonychium réparées si nécessaire. La tablette fenêtrée était ensuite repositionnée. Toutes les autres lésions ont été explorées sous anesthésie intrathécale et garrot de doigt. La tablette était retirée et les lésions du lit ou de la matrice réparées au $\operatorname{PDS}^{\circledR} 6 / 0$. La tablette était repositionnée ou, si elle était détruite, remplacée par la chambre d'une tubulure de perfusion (Fig. 2). Aucun antibiotique n'était donné.
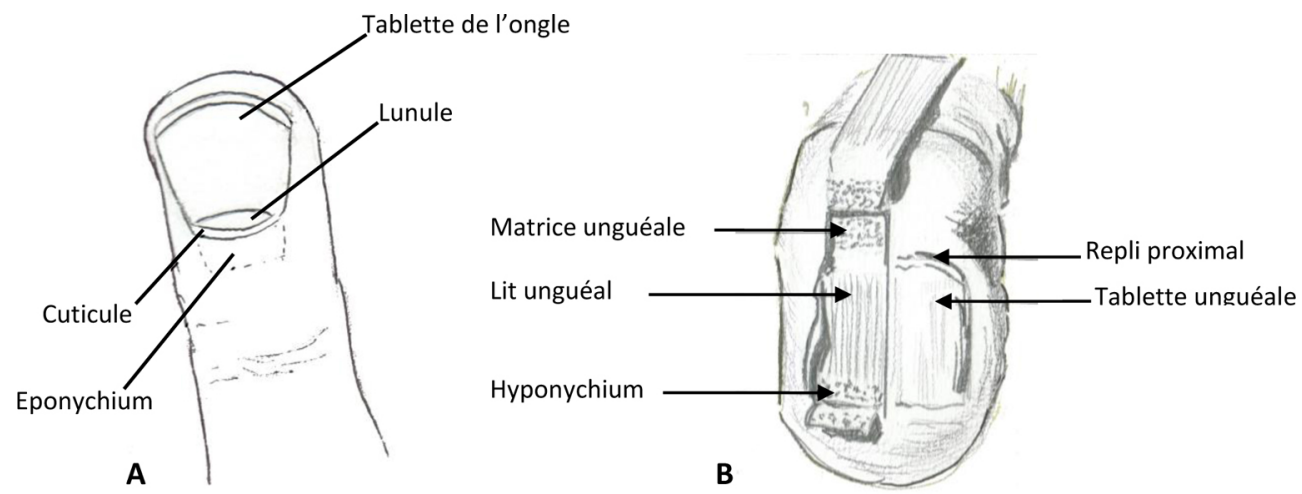

Fig. 1 Rappels anatomiques. A. La tablette unguéale est insérée dans les replis latéraux et proximaux (appelé aussi éponychium). La tablette est « scellée » au repli par la cuticule. Par transparence, on voit parfois (toujours sur le pouce) une convexité plus blanche appelée la lunule qui correspond à la limite distale de la matrice unguéale. B. Quand on retire la tablette, on aperçoit le lit unguéal qui présente des rainures longitudinales qui s'entrecroisent avec celles de la tablette et expliquent l'adhérence. Il n'y a pas d'adhérence entre la tablette et la matrice, ce qui permet l'expansion des hématomes sous-unguéaux. À la partie distale du doigt, la tablette perd son adhérence au niveau de l'hyponychium 


\section{Analyse statistique}

Les moyennes sont exprimées avec leur écart-type. S'agissant d'une étude épidémiologique, les données sont exprimées en valeur absolue et en pourcentage.

\section{Résultats}

Les traumatismes unguéaux étaient très fréquents dans notre unité et représentaient $8 \%$ des traumatismes digitaux. Sur 18 mois consécutifs, nous avons retrouvé 187 patients, dont 138 hommes (74\%) d'âge moyen de $38 \pm 25$ ans et 49 femmes (26\%) d'âge moyen de $46 \pm 19$ ans. Le côté droit était atteint dans 80 cas (43\%) et le gauche 106 fois (57\%), les données étaient manquantes dans un cas. Les patients se blessaient la main dominante dans $37 \%$ des cas. Le pouce était le doigt le plus souvent atteint avec 55 cas (29\%), suivi par le majeur dans 43 cas (23\%). Venaient ensuite l'index (31 cas, $17 \%$ ), l'annulaire (28 cas, $15 \%$ ) et l'auriculaire (19 cas, $10 \%$ ). Dans un dossier, le doigt atteint n'était pas précisé. Dans $5 \%$ des cas (dix patients), il y avait plusieurs doigts atteints et alors le majeur était toujours impliqué.

Il s'agissait d'un mécanisme d'écrasement le plus souvent (83 cas, $44 \%$ ), avec un doigt porte dans 42 cas (23\%) et un doigt écrasé par un objet dans 41 cas $(22 \%)$. Nous avons observé 55 lésions franches $(30 \%)$ parmi lesquelles un tiers était des accidents de machette, un outil traditionnel dans notre île. D'autres mécanismes ont été observés dans 35 cas. Les données étaient manquantes dans 14 cas.

Il s'agissait d'un accident de travail dans 82 cas (44\%), les travailleurs manuels étant les plus représentés (69 cas, $37 \%$ ). Venaient ensuite les employés et les fonctionnaires dans 26 cas, les retraités dans 16 cas, les apprentis dans 11 cas, divers emplois dans 36 cas et 12 chômeurs. Dans 19 cas, cette information était manquante.

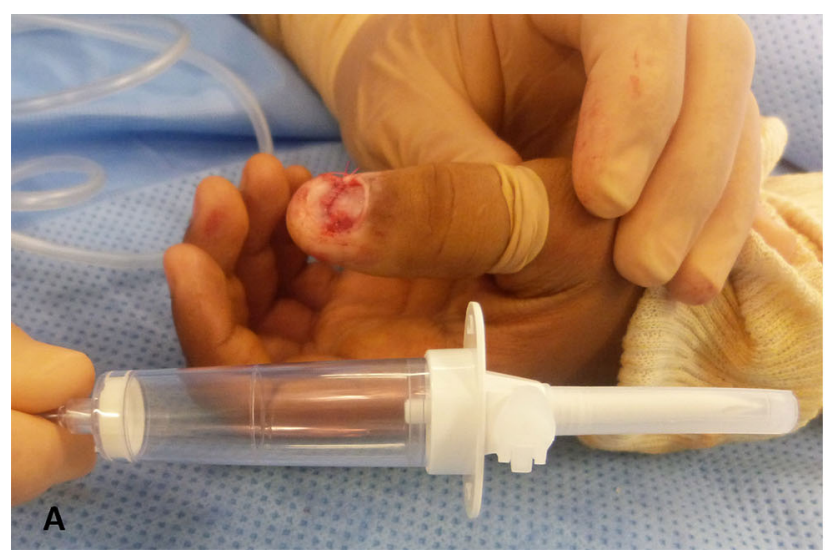

Dans 134 cas $(72 \%)$, il existait une lésion associée à la lésion unguéale. Une lésion pulpaire dans 50 cas (27\%), une fracture de la phalange distale dans 29 cas (16\%) et une association des deux lésions dans 49 cas (26\%). Une autre lésion plus proximale sur le doigt était notée dans 22 cas (12\%). La lésion associée siégeait sur le même doigt dans 128 cas.

Lors de l'exploration des lésions, nous avons retrouvé 47 lésions isolées de la tablette unguéale (25\%), 103 lésions du lit de l'ongle (55\%), 14 lésions de la matrice (parfois associées à des lésions du lit) et 23 hématomes sousunguéaux (12\%).

\section{Discussion}

Dans cette série monocentrique, nous avons pu confirmer certaines données de la littérature, mais d'autres n'ont pas été retrouvées. Dans notre série, l'âge des patients était plus élevé que dans les autres séries de la littérature dont l'âge moyen était inférieur à 30 ans [5,6], mais notre série n'incluait pas d'enfants de moins de 15 ans qui, pour des raisons réglementaires, étaient pris en charge dans un hôpital d'enfant.

La prédominance masculine est confirmée. Par contre, nos patients se blessaient plus souvent la main non dominante, contrairement aux autres séries, peut-être parce que nous avons plus de lésions par objet tranchant ou contondant et tenu dans la main dominante que par écrasement que les autres séries [3,7]. Les lésions unguéales font surtout suite à des écrasements, $44 \%$ dans notre série et $60 \%$ dans celle de Zook et al. [5], mais les lésions franches étaient aussi largement représentées, un biais possible étant lié à l'usage fréquent d'une machette dans notre région agricole.

Dans les séries publiées, le majeur était le doigt le plus atteint (à cause de sa longueur), alors que le pouce et l'auriculaire étaient les moins atteints [5,7]. Dans notre série,

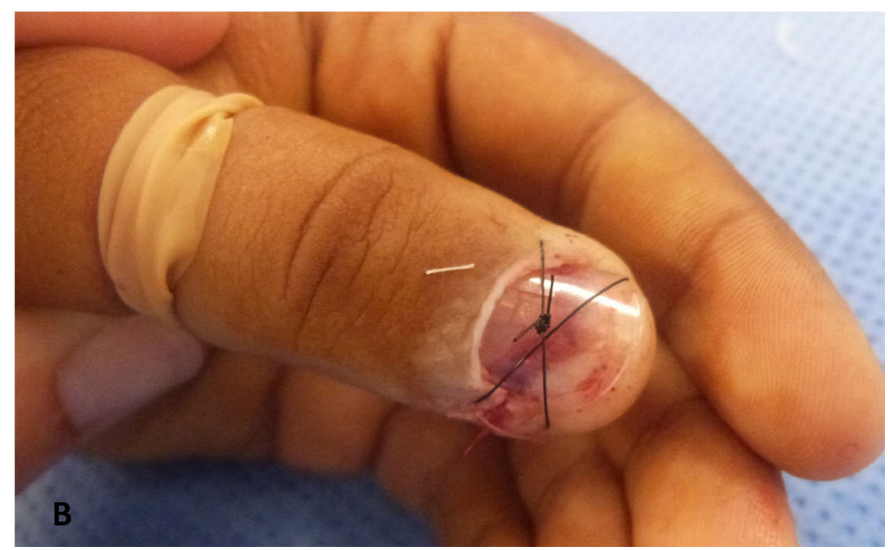

Fig. 2 Quand la tablette unguéale était détruite, nous utilisions pour la remplacer le piège à bulles d'une tubulure de perfusion. A. Le piège à bulles utilisé après la réparation du lit unguéal. B. Résultat après fixation par un point en $\mathrm{X}$ ne traversant pas la (néo) tablette pour ne pas fragiliser les réparations 
comme dans celle de Guy [6], c'était le pouce qui était le plus souvent atteint. Là encore, cela peut être lié à la différence entre écrasement (du doigt le plus long) et plaie (du pouce tenant l'objet).

Les lésions unguéales associées à d'autres lésions sont plus fréquentes que les lésions isolées. Ces lésions multiples représentent $80 \%$ des cas dans la série de Zook et al. comme dans la nôtre [5]. La tablette unguéale ayant un rôle protecteur, les lésions du périonychium supposent un mécanisme d'écrasement, ce qui entraîne une lésion du plancher (la phalange) et du plafond (la tablette). Nous avons observé $42 \%$ de lésions osseuses associées, alors que d'autres auteurs rapportent entre 50 et $60 \%$ de lésions osseuses, mais ils n'incluaient pas les lésions moins graves que sont les hématomes sous-unguéaux et les lésions isolées de la tablette dans leurs séries [5-7].

Nous avons choisi d'inclure dans notre étude les hématomes sous-unguéaux et les lésions isolées de la tablette qui n'apparaissent pas dans les séries chirurgicales ou sont exclues des séries chirurgicales, car elles ne sont pas vues/ traitées par les chirurgiens [8]. Elles sont pourtant fréquentes, représentant $37 \%$ de nos patients, et elles sont également consommatrices de temps.

Les lésions isolées de la tablette sont les moins graves des lésions unguéales et se pose la question de savoir si la tablette doit être reposée. Chez l'enfant, la question est très débattue, car on pense que cela augmente le risque infectieux [10]. O'Shaughnessy et al. n'ont trouvé aucune différence selon que la tablette était ou non remise en place [7], mais la plupart des auteurs continuent de recommander sa remise en place (ou un substitut), considérant que ne pas la remettre augmentait le risque de mauvais résultat sur la repousse unguéale en majorant le risque de dystrophie post-traumatique $[5,8,11,12]$. Souvent, ce n'est qu'au cours de l'exploration et après avoir soulevé la tablette qu'on s'aperçoit que la lésion est isolée [12], et il est selon nous logique de reposer la tablette pour diminuer les douleurs lors des pansements, protéger une éventuelle réparation du lit ou de la matrice, mouler la réparation des structures souvent contuses et éviter la fermeture du repli proximal source de ptérygion [13].

Il existe également un débat pour savoir comment prendre en charge les hématomes sous-unguéaux [11,14]. Seuls les hématomes sous-unguéaux les plus importants présentent des lésions du lit qui sont réparables [15], mais les travaux prospectifs considèrent qu'une exploration chirurgicale n'est peut-être pas justifiée [16].

Notre recueil ne peut être exhaustif, et les dossiers ont été revus de façon rétrospective. Cependant, seul médecin urgentiste accueillant les urgences de la main de l'île, nous avons probablement vu et suivi la très grande majorité des lésions unguéales. Ces lésions unguéales sont fréquentes. Le médecin urgentiste doit les connaître et ne pas les sousestimer, car dans plus de trois quarts des cas, elles nécessitent un geste technique qui relève le plus souvent de la compétence de l'urgentiste.

Remerciements Au Pr Christian Dumontier (Centre de la Main, Baie-Mahault, Guadeloupe), pour son aide à cette publication

Aux infirmières et aides-soignantes de l'unité de traumatologie de la main qui m'ont aidée à prendre en charge les patients

Liens d'intérêts : l'auteur déclare n'avoir aucun lien d'intérêt et n'avoir reçu aucun financement pour ce travail / I hereby declare that I have no conflicts of interest to disclose.

\section{Références}

1. Ardouin T, Poirier P, Rogez JM (1997) Fingertips and nailbed injuries in children. Apropos of 241 cases. Rev Chir Orthop Reparatrice Appar Mot 83:330-4

2. Duthie G, Adams J (1984) Meshed adhesive tape for the treatment of crushed fingers in children. J Hand Surg Br 9:41

3. Inglefield CJ, D'Arcangelo M, Kolhe PS (1995) Injuries to the nail bed in childhood. J Hand Surg Br 20:258-61

4. Shepard GH (1990) Management of acute nail bed avulsions. Hand Clin 6:39-58

5. Zook EG, Guy RJ, Russell RC (1984) A study of nail bed injuries: causes, treatment, and prognosis. J Hand Surg Am 9:247-52

6. Guy RJ (1990) The etiologies and mechanisms of nail bed injuries. Hand Clin 6:9-19 (discussion 21)

7. O’Shaughnessy M, McCann J, O'Connor TP, et al (1990) Nail regrowth in fingertip injuries. Ir Med J 83:136-7

8. Lorea P (2013) Primary care of nail traumas. Chir Main 32:129-35

9. Chevaleraud E, Ragot JM, Brunelle E, et al (1993) Anesthésie locale des doigts par la gaine des fléchisseurs. Ann Fr Anesth Reanim 12:237-40

10. Greig A, Gardiner MD, Sierakowski A, et al (2017) Randomized feasibility trial of replacing or discarding the nail plate after nailbed repair in children. Br J Surg 104:1634-9

11. Sommer NZ, Brown RE (2011) The perionychium. In: Wolfe SW, Hotchkiss RN, Pederson WC, Kozin SH (eds) Green's Operative Hand Surgery, Churchill Livingstone, Philadelphia, pp 333-53

12. Cheung K, Hatchell A, Thoma A (2013) Approach to traumatic hand injuries for primary care physicians. Can Fam Physician 59:614-8

13. Carmès $S$, Dumontier C (2014) Traumatismes de l'appareil unguéal (44-400). In: Techniques chirurgicales Orthopédie-Traumatologie, EMC, Paris, 1-11

14. Dean B, Becker G, Little C (2012) The management of the acute traumatic subungual haematoma: a systematic review. Hand Surg $17: 151-4$

15. Simon RR, Wolgin M (1987) Subungual hematoma: association with occult laceration requiring repair. Am J Emerg Med 5:302-4

16. Seaberg DC, Angelos WJ, Paris PM (1991) Treatment of subungual hematomas with nail trephination: a prospective study. Am J Emerg Med 9:209-10 